\title{
High-dose enzyme replacement therapy attenuates cerebroventriculomegaly in a mouse model of mucopolysaccharidosis type II
}

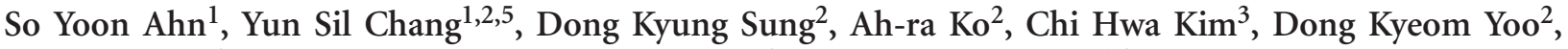 \\ Keun Ho Lim², Young Bae Sohn ${ }^{4}$, Dong Kyu Jin ${ }^{1,2}$ and Won Soon Park ${ }^{1,2}$
}

The natural progression of the severe form of mucopolysaccharidosis II in children is a rapid decline of neurodevelopmental function with hydrocephalus. Recombinant human iduronate-2-sulfatase enzyme replacement therapy (ERT) under a standard regimen seems to have limited effect. Therefore, we determined whether early, high-dose ERT attenuated ventriculomegaly and histologic abnormalities in the brains of IdS-knockout mice. IdS-knockout mice received saline or recombinant human IdS $\left(0.5 / 1.0 / 2.0 \mathrm{mg} \mathrm{kg}^{-1}\right)$ intravenously once weekly, starting at 4 weeks of age and continuing until 20 weeks. ERT with $2.0 \mathrm{mg} \mathrm{kg}^{-1}$, but not 0.5 or $1.0 \mathrm{mg} \mathrm{kg}^{-1}$, significantly attenuated enlarged ventricles, as confirmed by in vivo 7-teslar brain magnetic reasonance image (MRI) at 20 weeks. However, neuronal cytoplasmic vacuolization and morphological alteration in the purkinje cells on brain histology and glycosaminoglycan (GAG) levels in brain homogenates were reduced in mice receiving ERT at lower dose than $2.0 \mathrm{mg} \mathrm{kg}^{-1}$. Additionally, GAG levels significantly correlated with the percent volume ratio of ventricle to whole brain. These results suggested that high-dose systemic ERT started early in life could be a promising therapeutic modality for improving neurologic dysfunction including ventriculomegaly in children with severe Hunter syndrome. Journal of Human Genetics (2013) 58, 728-733; doi:10.1038/jhg.2013.92; published online 5 September 2013

Keywords: Hunter syndrome; MPS II mouse model; iduronate-2-sulfatase; brain disease; enzyme replacement therapy

\section{INTRODUCTION}

Mucopolysaccharidosis type II (MPS II, Hunter syndrome) is an $\mathrm{X}$-linked lysosomal storage disorder caused by deficiency of iduronate-2-sulfatase (IdS; EC 3.1.3.13), which is involved in the degradation of lysosomal glycosaminoglycan (GAG). ${ }^{1}$ Two clinical phenotypes exist, attenuated and severe, defined by the presence of neurologic involvement. The severe form displays progressive somatic and neurological involvement including mental retardation, hearing loss and ventriculomegaly. ${ }^{2}$ We previously reported a novel mouse model: IdS-knockout (KO) mice generated by disruption of the IdS locus through deletion of exons 2 and $33^{3-5} \mathrm{IdS}$-KO mice demonstrate distinct cellular vacuolization in the brain and a general phenotype that is consistent with the characteristics of the severe form of MPS II in humans.

A previous report on the natural progression of MPS II in children showed neural parenchymal disease with a rapid decline in cognitive and adaptive ability, and speech and language functions within 48 months of age. After that, impaired neurological function is maintained in a plateau phase without further improvement. ${ }^{6}$
CNS defects are devastating and important problems that must be resolved for quality-of-life reasons in patients with the severe form of Hunter syndrome. Intravenous infusion of recombinant human IdS (rhIdS) is effective in reducing lysosomal GAG and organ size, and improving walking ability and pulmonary function. ${ }^{7}$ However, no improvement in brain imaging abnormalities including white matter abnormalities, dilated perivascular spaces and ventriculomegaly have been observed in MPS II patients who receive enzyme replacement therapy (ERT) ${ }^{8}$ This limitation is partially thought to originate from inadequate enzyme uptake across the blood-brain barrier. Intraventricular administration demonstrated favorable corrective effects on CNS storage. ${ }^{9}$ However, to treat both visceral organs and the brain simultaneously, systemic rather than local recombinant human IdS delivery is the preferred ERT method. Addtionally, access to ventricular injection is limited in clinical settings. Thus, systemic ERT at high doses was investigated using an adult mouse model of MPS II $^{10}$ demonstrating that systemically delivered enzyme can cross the blood-brain barrier and allowed the possibility of high-dose ERT to protect CNS defects in Hunter syndrome. ${ }^{10}$ In the previous human

${ }^{1}$ Department of Pediatrics, Samsung Medical Center, Sungkyunkwan University School of Medicine, Seoul, Korea; ${ }^{2}$ Samsung Biomedical Research Institute, Sungkyunkwan University School of Medicine, Seoul, Korea; ${ }^{3}$ Rare disease therapeutics team, Mogam Biotechnology Research Institute, Yongin, Korea and ${ }^{4}$ Department of Medical Genetics, Ajou University School of Medicine, Suwon, Korea

${ }^{5}$ Co-first author.

Correspondence: Professor DK Jin, Department of Pediatrics, Samsung Medical Center, Sungkyunkwan University School of Medicine, 50 Irwon-dong, Gangnam-gu, Seoul 135 710, Korea. 
study, MPS II patients with cognitive impairment showed significantly higher ratio of lateral ventricles volume to intracranial volume than those with normal cognition, and this ratio of lateral ventricles volume to intracranial volume was accepted as an indicator of subcortical atrophy in the MPS II patients. ${ }^{11}$ To our knowledge, despite the clinical significance, the therapeutic effects of ERT in ventricular dilatation ${ }^{2,11}$ has not yet been evaluated in an animal model of Hunter syndrome. We therefore determined whether highdose systemic ERT, initiated early, at 4 weeks after birth in IdS-KO mice, attenuated ventriculomegaly and histologic abnormalities at 20 weeks after birth. We also determined whether the effects of IdS ERT on the CNS were dose dependent. In addition, cerebral ventriculomegaly was assessed for the first time in IdS-KO mice by in vivo brain magnetic reasonance imaging (MRI).

\section{MATERIALS AND METHODS}

\section{Animal model}

As previously reported, an IdS-KO mice were developed by Jin. ${ }^{3-5}$ Briefly, IdS$\mathrm{KO}$ mice were generated by replacing exons 2 and 3 of the IdS gene with a neomycin-resistance gene. Carrier females were bred with male mice from a B6/129 background strain. Offspring were heterogeneous females and hemizygous male KO mice, as well as wild-type (WT) males and females. The genotype of all mice used in experiments was confirmed by polymerase chain reaction of DNA from a tail snip. Experimental protocols were reviewed and approved by the Animal Care and Use Committee of Samsung Biomedical Research Institute, Seoul, Korea. This study was performed in accordance with institutional and National Institutes of Health guidelines for laboratory animal care.

Humidity was maintained at $50 \%$ and environmental temperature was $24^{\circ} \mathrm{C}$. Experiments began at 4 weeks after birth and continued through 20 weeks. Mice were allocated into five experimental groups: WT control (WT, $n=6)$, IdS-KO control (KO, $n=10$ ) and IdS-KO with IdS injection at $0.5,1.0$ or $2.0 \mathrm{mg} \mathrm{kg}^{-1}(\mathrm{KO}+\mathrm{IdS} 0.5, n=9 / \mathrm{KO}+\mathrm{IdS} 1.0, n=6 / \mathrm{KO}+\mathrm{IdS} 2.0, n=8)$. Recombinant human IdS enzyme (Elaprase, Shire, Cambridge MA, USA) was injected once a week into the lateral tail vein of mice, and equal volume of normal saline was injected in an identical manner to the WT and KO control groups. At 20 weeks, brain MRIs were taken, and mice were sacrificed by deep pentobarbital anesthesia $\left(60 \mathrm{mg} \mathrm{kg}^{-1}\right.$, intraperitoneal). Transcardiac perfusion was with ice-cold phosphate buffered saline (PBS), and skulls were removed in situ. Harvested whole-brain tissue was snap-frozen in liquid nitrogen and stored at $-80{ }^{\circ} \mathrm{C}$ until biochemical analyses. For morphometric analyses, whole brains were fixed with $4 \%$ paraformaldehyde overnight at room temperature.

In vivo magnetic reasonance imaging. Brain MRI used a 7.0-Tesla MRI system (Bruker-Biospin, Fällanden, Switzerland) under inhaled anesthesia with 1.5$2 \%$ isoflurane into $\mathrm{O}_{2}$-enriched air. MRI was with a 20 -cm gradient set capable of a rising time of $400 \mathrm{mTm}^{-1}$. Nine slices with $1.0-\mathrm{mm}$ thickness were obtained using the following parameters: for T2-weighted (T2W) images, $\mathrm{TR}=3000 \mathrm{~ms}, \mathrm{TE}=70 \mathrm{~ms}, \mathrm{FOV}=19.2 \mathrm{~mm} \times 19.2 \mathrm{~mm}$, matrix size $=192$ $\times 192, \mathrm{NEX}=6$. After MRI, rat pups were allowed to recover.

Assessment of the percent volume ratio of ventricles to whole brain from MRI. Nine coronal MRIs per mouse brain were obtained, and two examiners calculated the percent volume ratio manually by outlining serial images in a blinded manner using ParaVision 2.0.2 software (Bruker, BioSpin, Karlsruhe, Germany). The boundaries of ventricles or whole brains were outlined on each image, and the corresponding area was calculated using the software. Ventricles were well delineated, high-intensity areas in T2WI. Volume estimations were made according to the Cavalieri's principal, ${ }^{12}$ and the percent ratio of the total ventricle volume to the whole brain was used as the cerebral ventricular dilatation parameter in IdS-KO mice.

Histological analysis. For toluidine blue staining, brains were immersion-fixed in cold $2 \%$ paraformaldehyde with $2 \%$ glutaraldehyde in PBS, and the parietal neocortex was cut in $1 \mathrm{~mm}$ slices. Tissues were post fixed in $1 \%$ osmium tetroxide and embedded in Spurr's resin (Polysciences, Warrington, PA, USA). Sections were stained with toluidine blue and assessed using light microscopy for lysosomal storage, indicated by cytoplasmic vacuolization. The abnormal morphology of neurons, especially Purkinje cells in the cerebellum, was assessed with hematoxylin and eosin staining. For hematoxylin and eosin staining, after tissue processing, fixed brains were embedded in paraffin wax. Sections of $4 \mu \mathrm{m}$ were cut from paraffin blocks and stained with hematoxylin and eosin. Images of each section were captured with a magnifier digital camera using an Olympus BX40 microscope (Olympus Optical Co. Ltd., Tokyo, Japan) and saved as JPEG files.

TUNEL assay. Cell death in the periventricular area in the brain was evaluated with the immunofluorescent terminal deoxynycleotidyltransferasemediated deoxyuridine triphosphate nick end labeling (TUNEL) technique (kit S7110 ApopTag, Chemicon, Temecula, CA, USA). TUNEL-positive nuclei in the periventricular area was counted in a blinded manner. Three coronal sections $(+0.95 \mathrm{~mm}$ to $-0.11 \mathrm{~mm} /$ Bregma) were counted from each brain and three random non-overlapping fields from each section.

Preparation of brain tissue extracts for GAG analysis. Tissue extracts were prepared by homogenizing tissues in PBS using a tissue homogenizer. Homogenates were centrifuged at $20000 \times g$ for $30 \mathrm{~min}$, and supernatants were collected. Total protein concentration $\left(\mathrm{mg} \mathrm{ml}^{-1}\right)$ was assayed using the bicinchoninic acid (BCA) assay (Pierce, Rockford, IL, USA).

Quantitative analysis of GAG accumulation. GAG concentrations in tissue homogenates of brain were quantified by a colorimetric assay (Kamiya Biomedical Co., Seattle, WA, USA) according to the manufacturer's instructions, and absorbance was measured at $620 \mathrm{~nm}$ using a chondroitin 6-sulfate standard curve. GAG levels in tissue extracts were adjusted for protein concentration as determined using the BCA assay and expressed as $\mu \mathrm{g}$ GAG per mg protein.

Statistical analyses. Data were expressed as mean \pm s.e.m. For continuous variables with a normal distribution, a statistical comparison between groups was performed by one-way analysis of variance with Bonferroni's correction. A $P$-value of $<0.05$ was considered significant. Stata software (ver. 11.0, Stata Corp LP, College Station, TX, USA) was used for all analyses.

\section{RESULTS}

\section{Brain MRI}

Figure 1 shows representative MRI and ventricle sizes, defined as percent volume ratio of ventricle to whole brain, for each group at 20 weeks. Compared with the WT group, the KO group demonstrated significantly dilated ventricles that are usually observed in the severe type of Hunter syndrome, mainly on the lateral ventricles, but not on the 3 rd or 4 th ventricles (Figure 1a). Whereas other brain abnormal findings such as white matter abnormality or dilated perivascular space that can be observed in human patients with severe MPS $\mathrm{II}^{8}$ were not observed in Ids-KO mice. The percent volume ratio of ventricle to whole brain, which can be suggested as an indicator of subcortical atrophy, ${ }^{11}$ increased significantly in this Ids-KO mice compared with WT group. IdS replacement at $2.0 \mathrm{mg} \mathrm{kg}^{-1}$ $(\mathrm{KO}+\mathrm{IdS} 2.0)$ only significantly attenuated the volume ratio of ventricles to whole brain, but ERT at 0.5 or $1.0 \mathrm{mg} \mathrm{kg}^{-1}$ $(\mathrm{KO}+\mathrm{IdS} 0.5, \mathrm{KO}+\mathrm{IdS} 1.0)$ did not significantly reduced the ratio (Figures $1 \mathrm{~b}$ and $\mathrm{c}$ ).

\section{Histological analysis}

Figure 2a displays a representative brain histology of a parietal neocortex stained with toluidine blue and a cerebellum stained with hematoxylin and eosin for each group. Neuronal lysosomal storage (GAG) is presented as neuronal cytoplasmic vacuolization. ${ }^{13}$ The number of distinct and enhanced neuronal vacuolizations observed in the cortical area was significantly increased in the $\mathrm{KO}$ 


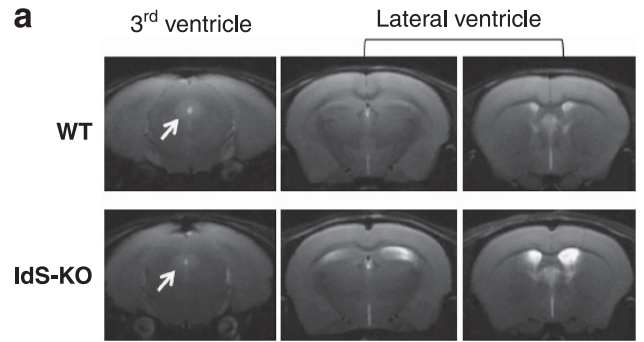

b

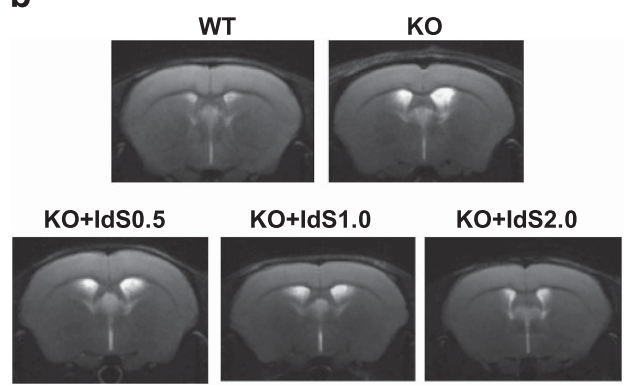

C

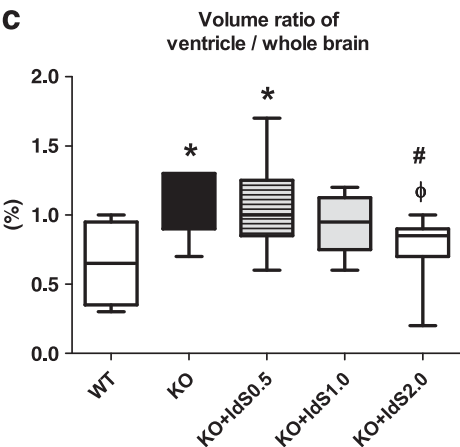

Figure 1 High-dose enzyme replacement therapy with iduronate-2-sulfatase (IdS) for 16 weeks reduced cerebroventricular dilatation in IdS-KO mice. (a) Representative brain magnetic reasonance images in wild-type and IdS-KO (knockout of iduronate-2-sulfatase) mice. IdS-KO mice display obviously dilated lateral ventricles, when compared with wild type. In the size of 3rd ventricle (white arrow), distinct difference between wild-type and IdS-KO mice was not observed. (b) Representative T2-weighted brain MRI performed at 20 weeks after birth in each group. (c) Significantly increased volume ratio of ventricle to whole brain in the $\mathrm{KO}$ group was attenuated in the $\mathrm{KO}+\mathrm{IdS} 2.0$ group, but not in the $\mathrm{KO}+\mathrm{IdSO} .5$ or $\mathrm{KO}+$ IdS 1.0 groups. Error bars mark the minimum and maximum values (range). WT, wild-type control group; KO, IdS-knockout control group; KO + IdSO.5; KO with IdS replacement at $0.5 \mathrm{mg} \mathrm{kg}^{-1} ; \mathrm{KO}+\mathrm{IdS} 1.0, \mathrm{KO}$ with IdS replacement at $1.0 \mathrm{mg} \mathrm{kg}^{-1} ; \mathrm{KO}+\mathrm{IdS} 2.0, \mathrm{KO}$ with IdS replacement at $2.0 \mathrm{mg} \mathrm{kg}^{-1} .{ }^{*} P<0.05 \mathrm{compared}$ with WT, ${ }^{\#} P<0.05$ compared with $\mathrm{KO},{ }^{\Phi} P<0.05$ compared with $\mathrm{KO}+\mathrm{IdSO} .5$.

group. IdS replacement at any dose $\left(0.5,1.0\right.$ or $\left.2.0 \mathrm{mg} \mathrm{kg}^{-1}\right)$ significantly ameliorated the neuronal lysosomal storage (Figures $2 \mathrm{a}$ and b). In the cerebellum, Purkinje cells displayed atypical morphology that might be related to the accumulation of GAG in the cerebellum $^{13}$ in the IdS-KO mice. ERT at the higher doses $(\mathrm{KO}+\mathrm{IdS} 1.0, \mathrm{KO}+\mathrm{IdS} 2.0)$, but not at $0.5 \mathrm{mg} \mathrm{kg}^{-1}$, seemed to show restoration of the morphological alterations of Purkinje cells in the MPS II mouse model (Figure 2a).

\section{Apoptosis in periventricular area \\ Apoptotic brain cell death in periventricular area was evaluated using TUNEL staining. When compared with WT, IdS-KO mice demon- strated significantly increased apoptosis evidenced by TUNEL-positive cells in the periventricular area (Figures $3 \mathrm{a}$ and b). Systemic ERT with $2.0 \mathrm{mg} \mathrm{kg}^{-1} \mathrm{IdS}$, which began at 4 weeks after birth and continued through 20 weeks, significantly reduced cell death in the periven- tricular area of the IdS-KO mice brain. No distinct difference in apoptosis was observed between $\mathrm{KO}$-IdS mice and $\mathrm{KO}$-IdS mice with ERT at $0.5 \mathrm{mg} \mathrm{kg}^{-1}$ or $1.0 \mathrm{mg} \mathrm{kg}^{-1}$ (data not shown). Moreover, there was a significant positive correlation between volume ratio of ventricles to whole brain and apoptosis in $\mathrm{WT}, \mathrm{KO}$ and $\mathrm{KO}+\mathrm{IdS} 2.0$ groups (Figure 3c).}

\section{GAG accumulation in the brain tissue}

IdS replacement in an IdS-KO mouse significantly reduced GAG levels in a dose-dependent manner (Figure 4). When compared with IdS treatment with $0.5 \mathrm{mg} \mathrm{kg}^{-1}(\mathrm{KO}+\mathrm{IdS} 0.5)$, IdS replacement at $1.0 \mathrm{mg} \mathrm{kg}^{-1}(\mathrm{KO}+\mathrm{IdS} 1.0)$ or $2.0 \mathrm{mg} \mathrm{kg}^{-1}(\mathrm{KO}+\mathrm{IdS} 2.0)$ was more effective at attenuating the GAG concentration in the brain tissue. No significant difference was observed between the $\mathrm{KO}+\mathrm{IdS} 1.0$ and
$\mathrm{KO}+\mathrm{IdS} 2.0$ groups (Figure 4a). Figure $4 \mathrm{~b}$ illustrates the correlation between brain GAG level and percent volume ratio of ventricle to whole brain in Ids-KO mice with or without ERT. The GAG concentration in the brain tissue significantly correlated with the percent volume ratio of ventricle to whole brain.

\section{DISCUSSION}

We confirmed for the first time by in vivo brain MRI that IdS-KO mice, a MPS II animal model, developed significant ventriculomegaly, which indicated by increased volume ratio of ventricles to whole brain, that resembled severe Hunter syndrome with hydrocephalus. In addition, we demonstrated that systemic weekly ERT, which started at 4 weeks after birth and maintained for 16 weeks, attenuated lysosomal storage in the brain, and ERT at the highest dose of $2.0 \mathrm{mg} \mathrm{kg}^{-1}$, but not at 0.5 or $1.0 \mathrm{mg} \mathrm{kg}^{-1}$, significantly attenuated the cell death in the periventricular area and ventriculomegaly, which assessed by in vivo brain MRI. In addition, the ventriculomegaly significantly correlated with the number of TUNEL-positive cells and GAG levels in brain tissue of IdS-KO mice. These findings suggested that increased volume ratio of ventricles to whole brain might be suggested as an associated marker of subcortical atrophy related with GAG accumulation in Hunter syndrome and implicated that high doses of IdS that exceed a certain critical level are required to improve cerebral atrophy in MPS II animals.

Although numerous previous reports used MPS II murine models, ${ }^{3-5,14,15}$ no animal study has evaluated ventricular dilatation or correlated its severity with other biological indicators of Hunter syndrome. Our study provides novel data on the effect on cerebral atrophy of systemic IdS replacement at a high dose, monitored by in vivo 7-teslar MRI. As ventriculomegaly is accepted as a critical 
a
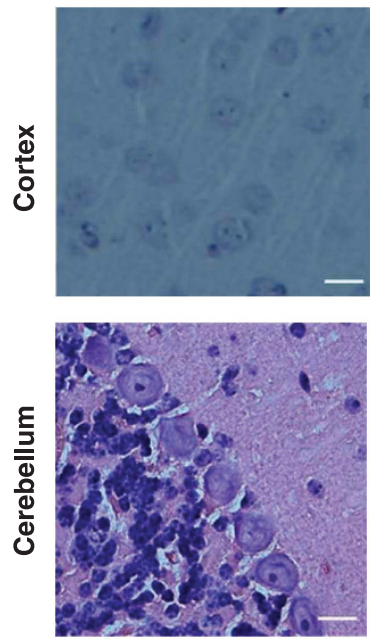

KO
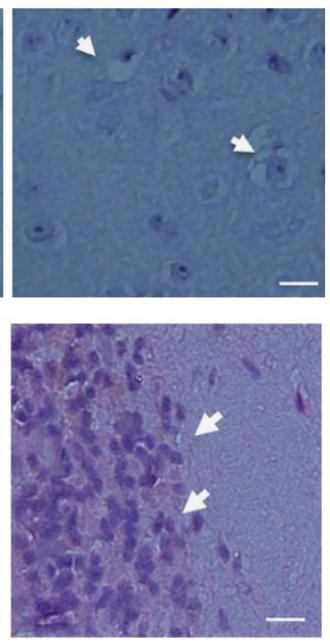

KO+ldS0.5
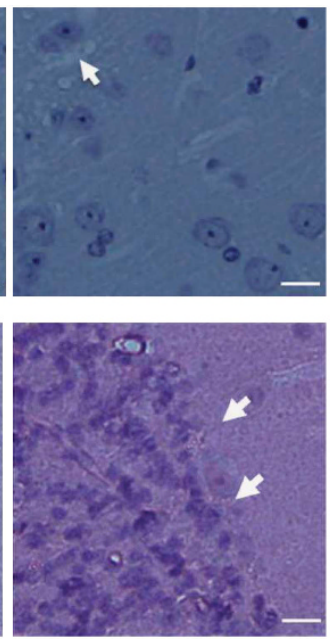

KO+IdS1.0
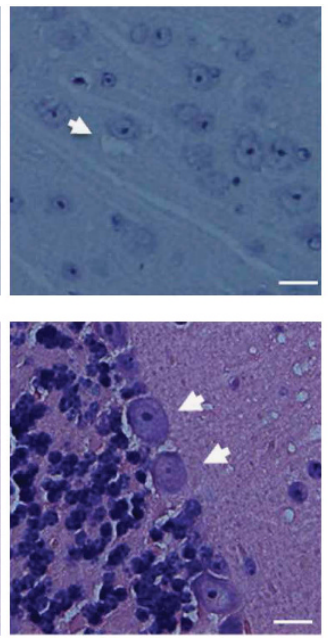

KO+ldS2.0
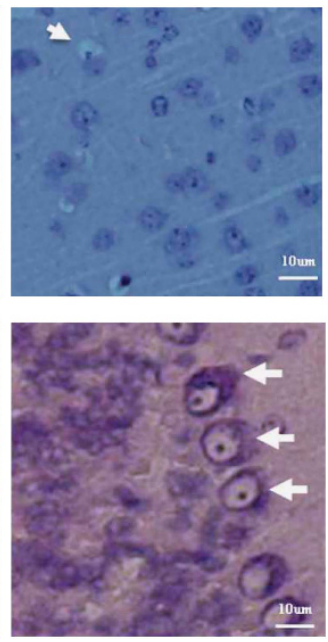

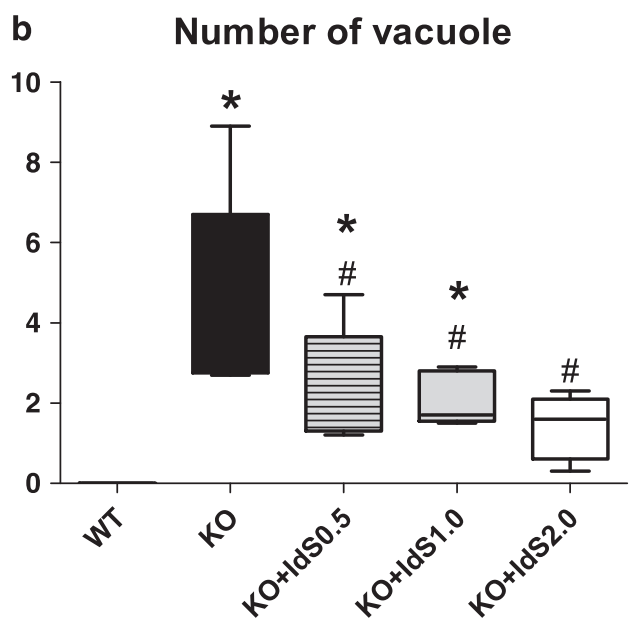

Figure 2 Enzyme replacement treatment with IdS for 16 weeks reduced lysosomal storage in the brain tissue of IdS-KO mice. (a) Representative optical microscopy photomicrographs of the cerebral cortex stained with toluidine blue and cerebellum stained with hematoxylin and eosin at 20 weeks after birth in each group (scale bars: $10 \mathrm{um}$ ). White arrows indicate cytoplasmic vacuoles in the lysosomal storage in neuronal cells in the parietal cortex and extensive morphological alteration of Purkinje cells in the cerebellum. (b) Significantly increased number of vacuoles in the KO group attenuated by enzyme replacement. No significant difference between ERT groups (KO + IdSO.5, KO IdS1.0 and KO + IdS2.0) was observed. Error bars mark minimum and maximum values (range). WT, wild-type control group; KO, IdS-knockout control group; KO+IdSO.5; KO with IdS replacement at $0.5 \mathrm{mgkg}^{-1}$; $\mathrm{KO}+\mathrm{IdS} 1.0, \mathrm{KO}$ with IdS replacement at $1.0 \mathrm{mg} \mathrm{kg}^{-1} ; \mathrm{KO}+\mathrm{IdS} 2.0$, KO with IdS replacement at $2.0 \mathrm{mg} \mathrm{kg}^{-1}$. ${ }^{*} P<0.05 \mathrm{compared}$ with $\mathrm{NC}$, \# $P<0.05$ compared with KO.

indicator of neurological impairment in children with MPS II, ${ }^{2,11}$ volumetric analysis of ventricles expressed by the fraction of whole brain with in vivo MRI in animals could be valuable as a clinically relevant tool in experimental MPS II research. Enlarged ventricles in Hunter syndrome are believed to be related to cortical atrophy or disruption of CSF reabsorption. In our previous report ${ }^{16}$ and in the present study, we showed apparently increased cell death in the periventricular area of the IdS-KO mice brain and demonstrated also significant positive correlation between cell death and volume ratio of ventricles to whole brain. Moreover, without dilatation of $3 \mathrm{rd}$ or 4 th ventricles, ventriculomegaly was observed mainly on the lateral ventricles of IdS-KO mice. Overall, ventricle enlargement in this IdS-KO mice model seems to be more related to the neurodegeneration and subcortical atrophy rather than interruption of CSF reabsorption, and we may suggest that high-dose ERT started in early childhood could ameliorate brain tissue damage in the periventricular area and improve the subcortical atrophy.
In a previous report on speech development in children with Hunter syndrome, hydrocephalus was observed in patients with the severe form and cognitive function declined as ventricles dilated. ${ }^{2}$ All children with MPS II who have hydrocephalus present with significant neurological complications including profound mental retardation and severely delayed speech. ${ }^{2}$ Thus, ventricular dilatation might be a representative indicator of impaired neurodevelopmental outcome in children with MPS II. ${ }^{11}$ Therefore, after the introduction of ERT for MPS II, questions about whether systemic ERT can reduce enlarged cerebroventricles have been raised. Wang et al. ${ }^{8}$ reported changes in brain imaging abnormalities of MPS I and II patients who were treated with ERT. In this study, only one MPS I patient, who received both ERT and hematopoietic stem cell transplantation, demonstrated attenuation of dilated ventricles. Another MPS I patient who was treated with ERT only showed improvement of white matter abnormalities without changes in dilated ventricles. ${ }^{8}$ In contrast, MPS II patients treated with 

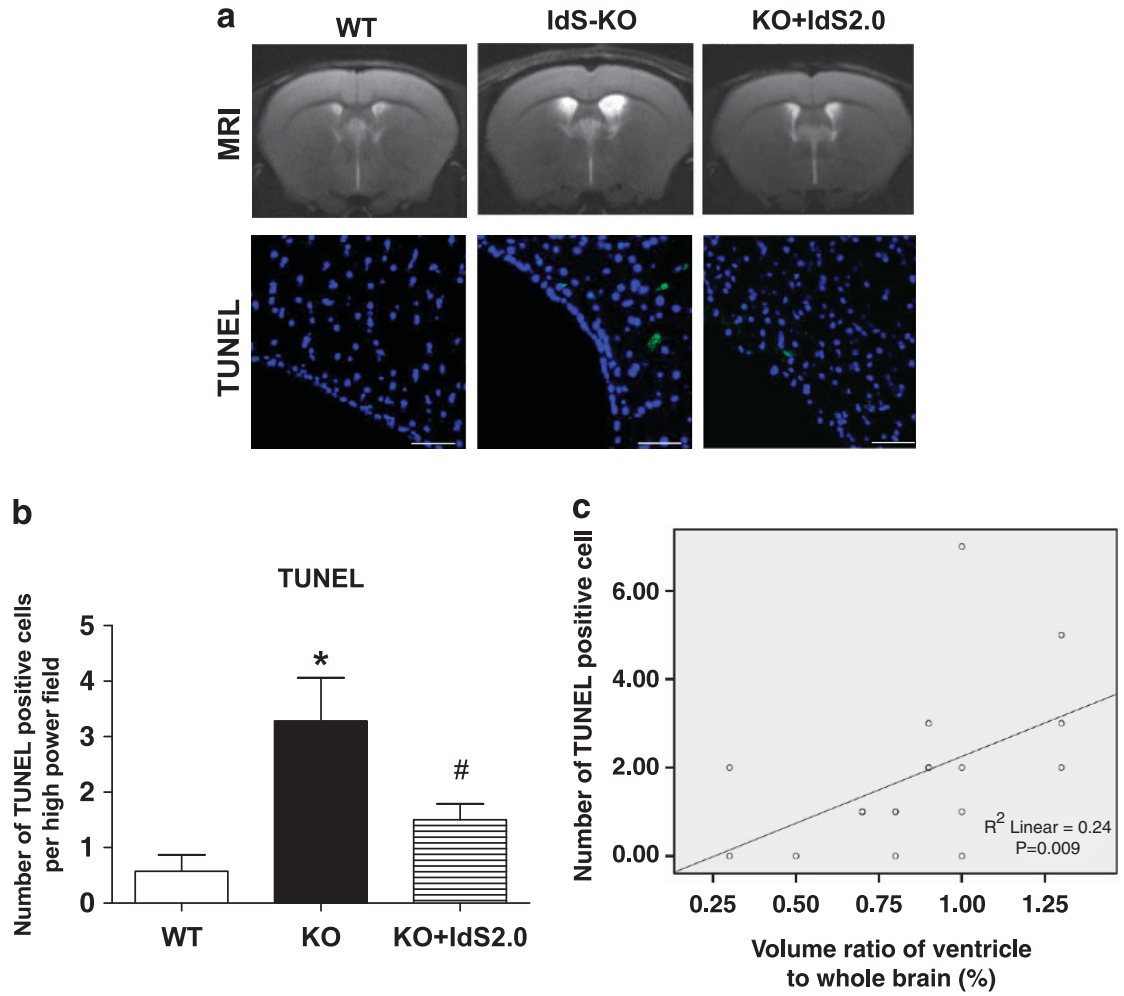

Figure 3 Increased cell death in the periventricular area of IdS-KO mice brain was decreased by high-dose enzyme replacement therapy. (a) Representative photomicrographs of the cell death in the periventricular area stained with TUNEL and representative brain MRI at 20 weeks after birth in KO, WT and KO + IdS2.0 groups. TUNEL were labeled with FITC (green), and the cell nuclei were labeled with DAPI (blue) (scale bars: 25 um). (b) The number of TUNEL-positive cells per high-power field in the periventricular area. (c) Significant positive correlation between the number of TUNEL-positive cells in the periventricular area and the volume ratio of ventricle to whole brain in WT, KO and KO+IdS2.0. Data are presented as the mean \pm s.e.m., WT, wild-type control group; $\mathrm{KO}$, IdS-knockout control group; $\mathrm{KO}+\mathrm{IdS} 2.0, \mathrm{KO}$ with IdS replacement at $2.0 \mathrm{mg} \mathrm{kg}^{-1}$. ${ }^{*} P<0.05$ compared with WT, \# $P<0.05$ compared with KO.
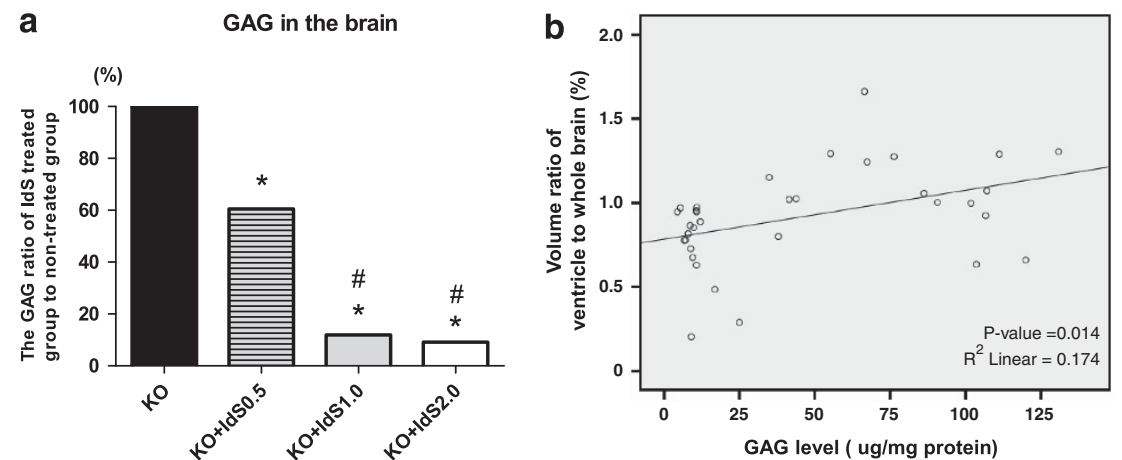

Figure 4 Lysosomal glycosaminoglycan (GAG) levels in brain tissue of IdS-KO mice decreased by enzyme replacement therapy. (a) KO group showed significantly increased GAG in the brain tissue, attenuated by enzyme replacement in a dose-dependent manner (without significance between the $\mathrm{KO}+\mathrm{IdS} 1.0$ and $\mathrm{KO}+\mathrm{IdS} 2.0$ groups). (b) Significant positive correlation between GAG level in the brain tissue and volume ratio of ventricle to whole brain was observed in $\mathrm{WT}, \mathrm{KO}, \mathrm{KO}+\mathrm{IdSO} .5, \mathrm{KO}+\mathrm{IdS} 1.0$ and $\mathrm{KO}+\mathrm{IdS} 2.0$ groups. WT, wild-type control group; $\mathrm{KO}$, IdS-knockout control group; $\mathrm{KO}+\mathrm{IdSO} .5$; $\mathrm{KO}$ with IdS replacement at $0.5 \mathrm{mg} \mathrm{kg}^{-1} ; \mathrm{KO}+\mathrm{IdS} 1.0, \mathrm{KO}$ with IdS replacement at $1.0 \mathrm{mg} \mathrm{kg}^{-1} ; \mathrm{KO}+\mathrm{IdS} 2.0, \mathrm{KO}$ with IdS replacement at $2.0 \mathrm{mg} \mathrm{kg}{ }^{-1}$. ${ }^{*} P<0.05$ compared with $\mathrm{KO},{ }^{\#} P<0.05$ compared with $\mathrm{KO}+\mathrm{IdSO} .5$.

standard-dose ERT did not present any changes in brain imaging abnormalities including white matter abnormalities, dilated perivascular spaces or ventriculomegaly. ${ }^{8}$ These findings correspond well with our results that showed no reduction in cell death in the periventricular area and enlarged ventricles in IdS-KO mice receiving standard doses of IdS $\left(0.5 \mathrm{mg} \mathrm{kg}^{-1}\right)$, compared with IdS-KO control mice. Unlike human patients with MPS II, in the present IdS-KO mice, it was difficult to observe other brain abnormal findings including white matter abnormality or dilated perivascular space. Thus, the therapeutic effects of systemic ERT for other brain abnormalities except ventriculomegaly could not be evaluated in the present study.

Vogler et al. ${ }^{13}$ reported that high-dose ERT for 13 weeks enabled the enzyme to be delivered across the blood-brain barrier and reduced lysosomal storage in the brain of mouse MPS VII models. 
Palito et al. ${ }^{10}$ showed correction of CNS defects via systemic ERT in an adult MPS II mouse model. However, in that study, the efficacy of ERT in improving neuronal density in older MPS II mice (7 months) was less than in younger mice ( 2 months) despite a high dose of ERT at $10 \mathrm{mg} \mathrm{kg}^{-1}$. When 2 -month-old mice received IdS at $1.2 \mathrm{mg} \mathrm{kg}^{-1}$ once every 4 days for 1 month, they showed a reduced urine GAG accumulation compared with normal WT mice and improved neurogenesis, reactive gliosis and cell death by brain histology. These results connoted that recovery from advanced neurodegeneration is limited, and, conversely, that initiation of ERT at a younger age for a longer period can lead to better resolution of CNS defects in people with MPS II. These findings coincide with our results. From 4 weeks of age, mice received IdS replacement at 0.5, 1.0 or $2.0 \mathrm{mg} \mathrm{kg}^{-1}$ for 16 weeks. This was effective in reducing GAG accumulation in the parietal neocortex in the MPS II mice. However, only the highest dose of ERT at $2.0 \mathrm{mg} \mathrm{kg}^{-1}$ effectively decreased cell death in the periventricular area and attenuated ventricular enlargement. This may suggest that early-started high-dose ERT may have a role in preventing GAG accumulation and ensuing brain damage, and this preventive approach may be more effective than corrective approach. At the same time, it may also indicate that GAG level should be reduced to a substantial degree in brain cells to induce protective effects in histopathology such as apoptosis or ventriculomegaly in IdS-KO mice. Therefore, our findings suggest that only high-dose ERT, above the standard dose, given early in life for a long period is a promising treatment regimen for improving neurologic impairment such as ventriculomegaly in clinically severe Hunter syndrome.

Our study has several limitations. First, our study did not conduct behavioral observations to evaluate memory, learning or sensorimotor functions. Further study is needed to determine whether high-dose systemic IdS ERT in IdS-KO mice is effective at not only resolving pathology such as ventriculomegaly but also improving brain function, which is the ultimate target of ERT for neurological manifestations of Hunter syndrome. Second, although our results indicated that a high dose of IdS exceeding a certain level is required to improved ventricular dilatation, ERT at doses higher than $2.0 \mathrm{mg} \mathrm{kg}^{-1}$ was not tested in our study. Recently, intraventricular ERT with a standard dose was reported to lead to stimulation of the enzyme in the brain and other tissues in a mouse model of Hunter syndrome. ${ }^{9,17}$ The intraventricular route was an excellent way for the IdS enzyme to cross the blood-brain barrier to reduce GAG levels in both the brain and other organs such as the liver, spleen and heart. ${ }^{9}$ To compare the efficacy of the administration route, estimation of the residual amount of IdS in the brain or other somatic organs after intravenous or intraventricular infusion would be needed. Third, a more mechanistic evaluation including brain IdS activity or cell death in reduction of ventriculomegaly needs to be explored in future studies. Moreover, we may need to consider the topic of antibody production related with systemic ERT. ${ }^{18}$ To figure out the doseresponse relation between the formation of anti- IdS antibodies and the injection dosage of IdS, the meticulously designed further study would be necessary.

In conclusion, high-dose $\left(2.0 \mathrm{mg} \mathrm{kg}^{-1}\right)$ systemic ERT with IdS for 16 weeks significantly attenuated ventricular dilatation. This was confirmed by in vivo brain MRI and effective reduction of lysosomal storage in the brain of IdS-KO mice. For clinical translation, a meticulously designed study to explore ERT regimens for optimal dosage, frequency and administration route is required.

1 Martin, R., Beck, M., Eng, C., Giugliani, R., Harmatz, P., Munoz, V. et al. Recognition and diagnosis of mucopolysaccharidosis II (Hunter syndrome). Pediatrics 121, e377-e386 (2008).

2 Cho, Y. S., Kim, J. H., Kim, T. W., Chung, S. C., Chang, S. A. \& Jin, D. K. Otologic manifestations of Hunter syndrome and their relationship with speech development. Audiol Neurootol 13, 206-212 (2008).

3 Jung, S. C., Park, E. S., Choi, E. N., Kim, C. H., Kim, S. J. \& Jin, D. K. Characterization of a novel mucopolysaccharidosis type II mouse model and recombinant AAV2/8 vector-mediated gene therapy. Mol. Cells. 30, 13-18 (2010).

4 Lee, J. H., Choe, Y. H., Kim, S. J., Paik, K. H. \& Jin, D. K. Changes in glycogen and glycosaminoglycan levels in hepatocytes of iduronate-2-sulfatase knockout mice before and after recombinant iduronate-2-sulfatase supplementation. Yonsei. Med. J. 52, 263-267 (2011).

5 Hong, S. H., Chu, H., Kim, K. R., Ko, M. H., Kwon, S. Y., Moon, I. J. et al. Auditory characteristics and therapeutic effects of enzyme replacement in mouse model of the mucopolysaccharidosis (MPS) II. Am. J. Med. Genet. A. 158A, 2131-2138 (2012).

6 Holt, J. B., Poe, M. D. \& Escolar, M. L. Natural progression of neurological disease in mucopolysaccharidosis type II. Pediatrics 127, e1258-e1265 (2011)

7 Muenzer, J., Wraith, J. E., Beck, M., Giugliani, R., Harmatz, P., Eng, C. M. et al. A phase $\mid \mathrm{I} / \mathrm{III}$ clinical study of enzyme replacement therapy with idursulfase in mucopolysaccharidosis II (Hunter syndrome). Genet. Med. 8, 465-473 (2006).

8 Wang, R. Y., Cambray-Forker, E. J., Ohanian, K., Karlin, D. S., Covault, K. K., Schwartz, P. H. et al. Treatment reduces or stabilizes brain imaging abnormalities in patients with MPS I and II. Mol. Genet. Metab. 98, 406-411 (2009).

9 Higuchi, T., Shimizu, H., Fukuda, T., Kawagoe, S., Matsumoto, J., Shimada, Y. et al. Enzyme replacement therapy (ERT) procedure for mucopolysaccharidosis type II (MPS II) by intraventricular administration (IVA) in murine MPS II. Mol. Genet. Metab. 107, 122-128 (2012).

10 Polito, V. A., Abbondante, S., Polishchuk, R. S., Nusco, E., Salvia, R. \& Cosma, M. P. Correction of CNS defects in the MPSII mouse model via systemic enzyme replacement therapy. Hum. Mol. Genet. 19, 4871-4885 (2010).

11 Fan, Z., Styner, M., Muenzer, J., Poe, M. \& Escolar, M. Correlation of automated volumetric analysis of brain MR imaging with cognitive impairment in a natural history study of mucopolysaccharidosis II. AJNR. Am. J. Neuroradiol. 31, 1319-1323 (2010).

12 Gundersen, H. J. \& Jensen, E. B. The efficiency of systematic sampling in stereology and its prediction. J. Microsc. 147, 229-263 (1987).

13 Vogler, C., Levy, B., Grubb, J. H., Galvin, N., Tan, Y., Kakkis, E. et al. Overcoming the blood-brain barrier with high-dose enzyme replacement therapy in murine mucopolysaccharidosis VII. Proc. Natl. Acad. Sci. USA 102, 14777-14782 (2005).

14 Garcia, A. R., Pan, J., Lamsa, J. C. \& Muenzer, J. The characterization of a murine model of mucopolysaccharidosis II (Hunter syndrome). J. Inherit. Metab. Dis. 30, 924-934 (2007).

15 Polito, V. A. \& Cosma, M. P. IDS crossing of the blood-brain barrier corrects CNS defects in MPSII mice. Am. J. Hum. Genet. 85, 296-301 (2009).

16 Sohn, Y. B., Lee, J., Cho, S. Y., Kim, S. J., Ko, A. R., Nam, M. H. et al. Improvement of CNS defects via continuous intrathecal enzyme replacement by osmotic pump in mucopolysaccharidosis type II mice. Am. J. Med. Genet. A. 161A, 1036-1043 (2013).

17 Garcia, A. R., DaCosta, J. M., Pan, J., Muenzer, J. \& Lamsa, J. C. Preclinical dose ranging studies for enzyme replacement therapy with idursulfase in a knock-out mouse model of MPS II. Mol. Genet. Metab. 91, 183-190 (2007).

18 Zareba, G. Idursulfase in Hunter syndrome treatment. Drugs of today 43, 759-767 (2007). 